\title{
Correction to: Cost-effectiveness analysis of rotavirus vaccination in China: Projected possibility of scale-up from the current domestic option
}

\author{
Shuhui Cui ${ }^{1}$, Ruoyan Gai Tobe ${ }^{1,2^{*}}$, Xiuting Mo ${ }^{1}$, Xiaoyan Liư ${ }^{1}$, Lingzhong Xu ${ }^{1}$ and Shixue $\mathrm{Li}^{1}$
}

\section{Correction}

After the publication of our article [1] we have been made aware of a number of mislabelling and reporting errors, which were introduced in the preparation of the manuscript. The conclusions are not affected by these errors and thus remain unchanged.

\section{The corrections required are as follows Correction 1}

In the Methods section under the heading "Vaccine effectiveness", the sentence:

"The protection effectiveness of Rotarix and Rotateq were derived from randomized controlled trials in other Asian regions such as Hong Kong, Taiwan and Singapore, considering the ethnic homogeneity [25], because there was no eligible data specifically for the Chinese population."

has been corrected to:

"The protection effectiveness of Rotarix and Rotateq were derived from randomized controlled trials [25] and clinical reviews cited by economic evaluation studies in other Asian regions such as Hong Kong, Taiwan and Singapore, due to no eligible data specifically for the Chinese population."

\section{Correction 2}

In the Results section under the heading "Health impacts and cost-effectiveness of vaccination", the sentence:

"The total cost is even less than non-vaccination."

has been corrected to:

"The ACER is even less than non-vaccination."

\footnotetext{
* Correspondence: gai-r@ncchd.go.jp

'School of Public Health, Shandong University, Jinan, China

${ }^{2}$ Department of Health Policy, National Center for Child Health and

Development, Okura 2-10-1, Setagaya-ku, Tokyo 157-8535, Japan
}

\section{Correction 3}

Four corrections are required to Table 1 as follows:

Rotateq efficacy: the plausible range for sensitivity analysis "0 - 0.98 " has been corrected to "0.883 - 1"; Source " 38 " has been corrected to " 38,42 ".

"Mortality rate" under the heading "Parameters" has been removed as it appeared twice in the Table.

Costs for international vaccinations: the plausible range for sensitivity analysis " $5-250$ " has been corrected to " 50 - 250 "

Infection rate: Source: "34" has been corrected to "21, 45". A corrected version of Table 1 appears below.

\section{Correction 4}

The values presented in Table 2 were mislabelled and incorrectly shown. The correct version of Table 2 is shown below.

\section{Correction 5}

The axes in Figure 2 were mislabelled. The correct version of Figure 2 is shown below.

\section{Correction 6}

The following reference should be included in the reference list:

45. Wu J, Yao Y, Hao W. Clinical Epidemiological Study on 244 Cases of Neonatal Rotavirus Infection. Chin J Nosocomiol, 1999, 9(4): 228-29 (in Chinese).

Received: 30 August 2018 Accepted: 30 August 2018

Published online: 27 September 2018

\section{Reference}

1. Cui S, Tobe RG, Mo X, Liu X, Xu L, Li S. Cost-effectiveness analysis of rotavirus vaccination in China: projected possibility of scale-up from the current domestic option. BMC Infect Dis. 2016;16(1):677. 
Table 1

\begin{tabular}{|c|c|c|c|c|}
\hline & Baseline & \multicolumn{2}{|c|}{ Plausible range for sensitivity analysis } & Sources \\
\hline \multicolumn{5}{|l|}{ Parameters } \\
\hline Discount rate & 0.03 & 0 & 0.03 & [31] \\
\hline Vaccine coverage & $25.3 \%$ & $10 \%$ & $28.6 \%$ & {$[36,37]$} \\
\hline Mortality rate & $0.0058 \%$ & 0.000029 & 0.000039 & [41] \\
\hline Rotateq efficacy & $98 \%$ & 0.883 & 1 & {$[38,42]$} \\
\hline Rotateq infected & $0.018 \%$ & 0 & 0.00018 & {$[42]$} \\
\hline hospitalization $1^{a}$ & $44 \%$ & 0 & 0.44 & {$[22]$} \\
\hline Outpatient1 $^{\text {a }}$ & $28 \%$ & 0 & 0.28 & {$[22]$} \\
\hline Home-care ${ }^{a}$ & $28 \%$ & 0 & 0.28 & {$[22]$} \\
\hline Rotarix infected & $0.1 \%$ & 0 & 0.001 & [26] \\
\hline LLR infected & $0.9 \%$ & 0 & 0.009 & {$[41]$} \\
\hline hospitalization $3^{c}$ & $0.2 \%$ & 0 & 0.002 & {$[2]$} \\
\hline Outpatient $3^{c}$ & $7.9 \%$ & 0 & 0.079 & [2] \\
\hline 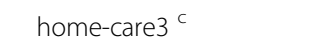 & $91.9 \%$ & 0 & 0.919 & {$[2]$} \\
\hline Rotarix efficacy & $96.1 \%$ & 0.871 & 1 & {$[25,26]$} \\
\hline LLR efficacy & $72 \%$ & 0.63 & 0.79 & {$[27]$} \\
\hline Infection rate & $78.85 \%$ & 0 & 0.7885 & {$[21,45]$} \\
\hline home-care2b & $32 \%$ & 0 & 0.32 & [22] \\
\hline hospitalization2 b & $33 \%$ & 0 & 0.33 & [22] \\
\hline Outpatient2 b & $35 \%$ & 0 & 0.35 & [22] \\
\hline natural protact1d & $77 \%$ & 0 & 0.77 & [23] \\
\hline natural protact2 d & $83 \%$ & 0 & 0.83 & [23] \\
\hline \multicolumn{5}{|l|}{ Costs } \\
\hline International vaccinations & 200.00 & 50 & 250 & {$[16,17]$} \\
\hline LLR vaccination & 24 & & & The national tariff \\
\hline Hospitalizations & 570.04 & 0 & 570.04 & {$[43]$} \\
\hline Outpatient & 104.19 & 0 & 104.19 & {$[43]$} \\
\hline Home-care & 11.52 & 0 & 11.52 & [44] \\
\hline \multicolumn{5}{|l|}{ Health Effects } \\
\hline QALY(Hospitalization) & 0.077 & 0.075 & 0.078 & {$[30]$} \\
\hline QALY(Outpatient) & 0.081 & 0 & 0.081 & {$[30]$} \\
\hline QALY(Home-care) & 0.082 & 0 & 0.082 & {$[30]$} \\
\hline
\end{tabular}

Table 2 Costs, health impacts and cost-effectiveness of rotavirus vaccines with comparison to no intervention

\begin{tabular}{llll}
\hline Strategy Name & Cost & QALYs & Incremental cost-effectiveness ratio $(\$ / Q A L Y)$ \\
\hline No vaccine & 2379.945 & 17.71296 & $(-)$ \\
LLR vaccine & 2507.851 & 22.65899 & 0 \\
Rotarix vaccination & 5982.187 & 24.31454 & 2105.66 \\
Rotateq vaccination & 5577.902 & 24.44506 & 1715.14 \\
\hline
\end{tabular}




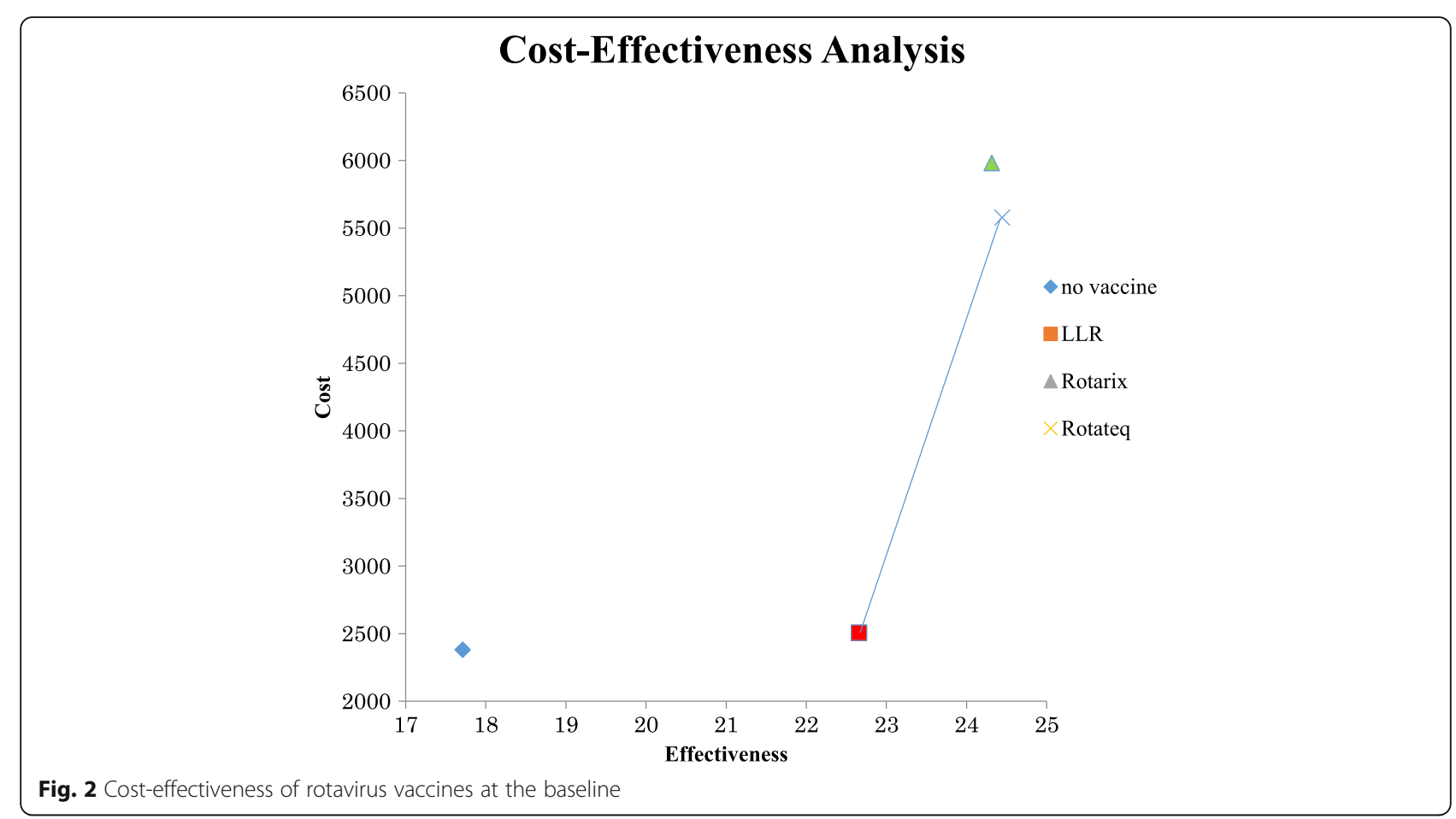

